\title{
An Integrated Platform for Differential Electrochemical and ISFET Sensing
}

\author{
Sara S. Ghoreishizadeh *, Pantelis Georgiou *, Sandro Carrara ${ }^{\dagger}$, and Giovanni De Micheli ${ }^{\dagger}$ \\ ${ }^{*}$ Centre for Bio-Inspired Technology, Imperial College London \\ ${ }^{\dagger}$ Integrated Systems Centre, École Polytechnique Fédérale de Lausanne (EPFL)
}

\begin{abstract}
A fully-integrated differential biosensing platform on CMOS is presented for miniaturized enzyme-based electrochemical sensing. It enables sensor background current elimination and consists of a differential sensor array and a differential readout IC (DiRIC). The sensor array includes a four-electrode sensor for amperometric electrochemical sensing, as well as a differential ISFET-based $\mathrm{pH}$ sensor to calibrate the biosensors. The ISFET is biased in weak inversion and co-designed with DiRIC to enable pH measurements from 1 to 14 with resolution of $0.1 \mathrm{pH}$. DiRIC enables differential current measurement in the range of $\pm 100 \mu \mathrm{A}$ with more than $120 \mathrm{~dB}$ dynamic range.

Index Terms-Sensor array, ISFET, differential readout
\end{abstract}

\section{INTRODUCTION}

Point-of-care testing increases the likelihood that the patient, physician, and care team will receive the results quicker, which allows for immediate clinical decisions to be made. Such testing requires biosensing platforms that are sensitive enough to the target metabolites, fast, autonomous, and ideally inexpensive. Integrating miniaturized biosensor arrays with CMOS instrumentation circuit introduces the opportunity for low-cost and mass-manufacturable sensing platform. The integration also improves the signal to noise ratio (SNR) of the measurements and provides scalability by removing the long wire interconnects.

Enzyme-based electrochemical sensors are commonly used to detect biomolecules such as glucose, lactate, and Adenosine triphosphate (ATP) in blood-testing in clinical laboratories. The enzymatic sensors provide good selectivity and fast and reliable measurements. An array of such sensors, each functionalized with a selective enzyme, enables a thorough study of different agents in a fluid such as human blood. Moreover, when integrated with CMOS circuit, they can provide inexpensive tools for patients at home to continuously monitor their metabolites.

Such integrated platforms have been introduced over the last decade for DNA analysers based on affinity-based sensors [1], [2]. The enzymatic-sensor miniaturization challenges include detection of small sensitivity and signal levels (in pico- or femto- ampere range) due to small sensor area. Nanostructures such as Carbon-nano-tubes (CNT) [3] or gold nanoparticles [1] are placed or grown on the sensor to increase the current level and sensitivity of the sensor. The nanostructures increase the effective area of the sensor by adding a three-dimensional structure to the sensor and improve enzyme immobilization.
However, most often the sensor background current also increases in presence of the nano-structures to typically much larger than the biomolecule-sensitive current. In the case of cyclic voltammetry, for example, this stems from large periodic background current from charging and discharging of the double layer capacitance at the electrode-electrolyte interface [4]. The presence of a large background current when reading out the small redox current requires a high resolution $\mathrm{ADC}$ and increases the power consumption of the sensor readout.

In this paper a differential sensing platform is presented on CMOS for miniaturized enzyme-based electrochemical sensing. The on-chip sensor and CMOS readout circuit are co-designed to enable small current measurement in presence of large background current. The system hence provides wide dynamic range in current detection and produces a digital output that in principle only depends on the target-biomolecule concentration. Amperometric electrochemical reactions such as cyclic voltammetry and chronoamperometry depend also on the $\mathrm{pH}$ of the solution [5]. In order to calibrate the biomolecular sensing with the $\mathrm{pH}$, an ion sensitive field effect transistor (ISFET) [6] is also embedded into the sensor array. Prior efforts in differential sensing include differential IC for amperometric DNA detection [7] in which charge generated at two electrodes are sampled at different phases of the clock and transformed into voltage with a switched-capacitor transimpedance amplifier. The difference of the two voltages is then calculated off-chip. In [8] a pseudo-differential readout IC is presented for multi-purpose electrochemical detection using off-chip components.

The design of the differential sensor array and the amperometric readout circuit are presented in the next section and a subsection is dedicated to ISFET design to comply with DiRIC and the $\mathrm{pH}$ sensing specifications. Simulation results are shown in Section IV followed by conclusions.

\section{DIFFERENTIAL SENSING PLATFORM}

\section{A. Differential sensor array}

An on-chip sensor array is designed to measure biomolecules such as glucose, lactate and ATP. The sensor core is a three electrode based electrochemical cell with a Working electrode $\left(W E_{\text {main }}\right)$ on which the nano-structure and the enzyme are immobilized, a Reference electrode (RE) and a counter electrode (CE). 


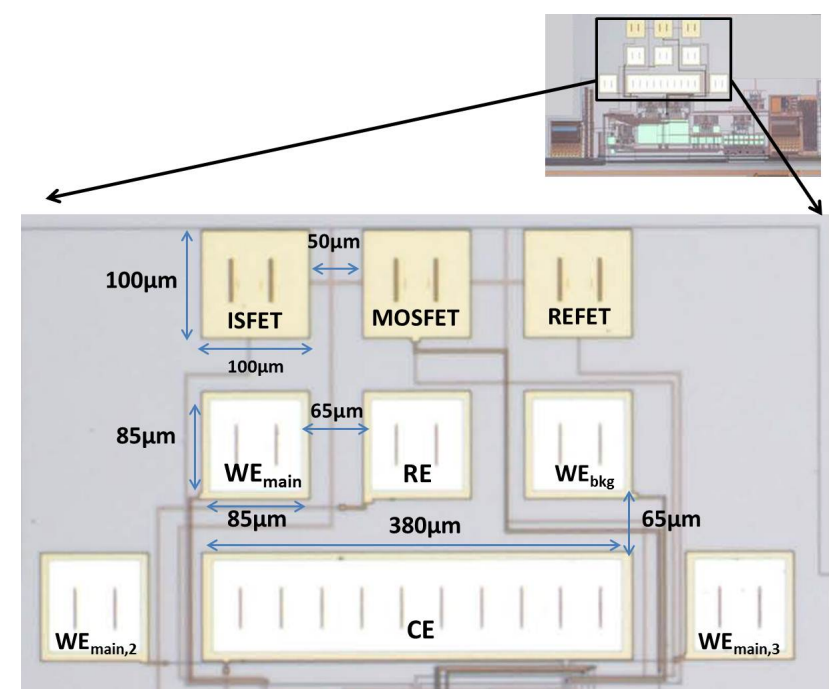

Fig. 1. The micro-photograph of integrated system where the on-chip differential sensor array is highlighted. The array includes $W E_{\text {main }}, W E_{b k g}, \mathrm{RE}$ and $\mathrm{CE}$, as well as an ISFET and REFET for differential $\mathrm{pH}$ measurement. $W E_{\text {main }, 2}, W E_{\text {main }, 3}$ are added for multiple-metabolite detection.

The current at the WE consists of a background current and a redox current. The background current doesn't change with the concentration of the target biomolecule and is partly due to the interferences from other electro-active molecules in the solution and in main part due to the current of the double-layer capacitor. Both components of the background current increase significantly in presence of nano-structures such as CNT where in particular the capacitive current increases. In this work a second WE $\left(W E_{b k g}\right)$ is added to the three-electrode cell to provide a duplicate of the background current. This can be achieved by covering the $W E_{b k g}$ with a nano-structure similar to $W E_{\text {main }}$ but without the selective-enzyme. The current of $W E_{b k g}$ is then deduced from the current of $W E_{b k g}$ with a readout-circuit resulting in solely redox current, which changes with target biomolecule's concentration.

The floorplan of the on-chip sensor array is shown in Fig. 1. The main cell includes two $85 \times 85 \mu \mathrm{m}$ WEs, a common RE, and a common CE. The $W E_{\text {main }}$ and $W E_{b k g}$ are placed symmetrical with respect to the RE and $\mathrm{CE}$ to ensure the resulted background current at the two WEs are equal. One single strand of CNTs can short-connect two electrodes if they are placed too close to each other. Therefore, the size and spacing of the electrodes are chosen considering the limitations of the post-processing techniques such as the electro-deposition of CNT and the enzyme immobilization. The CE is the largest electrode to reduce the CE electrodeelectrolyte resistance and consequently to prevent its floating voltage to reach the supply rails [3].

To study the effect of symmetry between $W E_{\text {main }}$ and $W E_{b k g}$ in accuracy of background current removal, two more working electrodes, $W E_{\text {main }, 2}$ and $W E_{\text {main }, 3}$, are placed asymmetrical with respect to RE and CE, compared to $W E_{b k g}$.

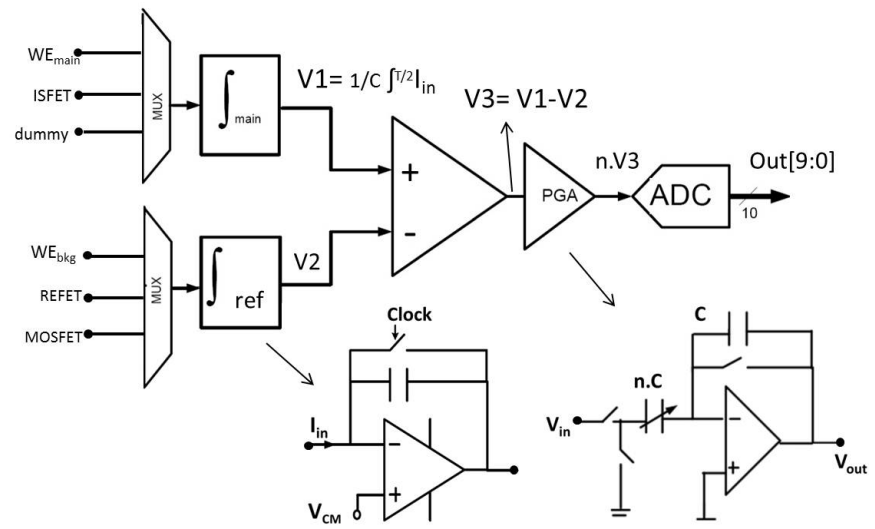

Fig. 2. The block diagram of DiRIC to measure and amplify the difference of two sensor currents. Two integrators at the front integrate the current of the sensors. The difference is amplified through the PGA.

\section{B. Differential Readout IC}

The schematic view of DiRIC is shown in Fig. 2. The circuit consists of four main building blocks:

(i) Two multiplexers select the sensors to be measured. The multiplexer at the main integrator connects it to one of the onchip $W E_{\text {main }}$, off-chip $W E_{\text {main }}$ or the drain of an ISFET. The multiplexer at the reference integrator connects it to one of the on-chip $W E_{C}$, off-chip $W E_{C}$, the drain of a REFET or a MOSFET. Dummy switches and structures are used to provide symmetry for the two integrators. For the sake of brevity, only few possible connections are shown in Fig. 2.

(ii) two identical integrators convert the sensor currents into voltages. The switch-capacitor(SC) based integrator is chosen as the first stage of the readout circuit because of two reasons: The sensor current (i.e. $I_{i n}$ in Fig. 2) is lowpass filtered straight away, which removes the high frequency noise in the electrochemical process; Moreover, by changing the reset clock of the integrator, input current with a wide dynamic range can be measured. The two integrators integrate the sensor current at the exact same time using the same clock signal. However, the accuracy of the readout is limited by the the noise and leakage of the op-amp and the switches in the integrators, which can be minimised by proper design and sizing of the devices.

(iii) A differential SC amplifier is designed similar to [9] to take the difference of the generated voltages at the output of the integrators. This block converts the differential voltage into a single one (with a unity-gain) and adjusts its output common-mode to the middle of supply voltage. This stage is where the effect of the background current is removed from the main current.

(iv) a programmable gain amplifier (PGA) amplifies the output of the differential SC amplifier. The PGA is realized in a SC architecture where the gain is proportional to the ratio of the capacitors (i.e. $n$ in Fig. 2). A programmable capacitor array is designed with four-bit dynamic-range. Therefore, the gain of the PGA can be programmed to any integer number from 1 to 16 . The output stage includes a 10 bit-SAR ADC 
TABLE I

SIMULATED SPECS. OF THE ISFET BIASED IN WEAK INVERSION

\begin{tabular}{cc}
\hline Parameter & Value \\
\hline$(W / l)_{\text {elec }}$ & $20 \mu \mathrm{m} / 10 \mu \mathrm{m}$ \\
$(W / l)_{\text {chem }}$ & $100 \mu \mathrm{m} / 100 \mu \mathrm{m}$ \\
$\operatorname{Min}\left(I_{\text {ISFET }}\right)$ & $16.4 \mathrm{nA}$ \\
$\operatorname{Max}\left(I_{\text {ISFET }}\right)$ & $1169 \mathrm{nA}$ \\
$V_{\text {RS }}$ & $1.55 \mathrm{~V}$ \\
\hline
\end{tabular}

and a serializer enabling voltages as small as $3.3 \mathrm{mV}$ to be resolved at the output of PGA.

\section{ISFET Design}

An ISFET is added to the sensor array for $\mathrm{pH}$ measurement in order to calibrate the biomolcular measurements (such as cyclic voltammetry) with the $\mathrm{pH}$. A REFET and a MOSFET with the exact same size are added to the sensor array to provide electrochemical and electrical references for the ISFET, respectively. The ISFET design goal is to enable $\mathrm{pH}$ measurements from 1 to 14 with resolution of $0.1 \mathrm{pH}$. The steps to find the electrical dimension of an ISFET that satisfies this requirement, when interfaced with DiRIC, are presented in this section.

The threshold voltage of the ISFET, $V_{t h}$ changes linearly with the $\mathrm{pH}$ of analyte in contact with:

$$
V_{t h}=V_{t h 0}+\alpha \times \Delta p H
$$

Where $V_{t h 0}$ is the threshold voltage of the device at a reference $\mathrm{pH}$ and $\Delta p H$ is the change in the $\mathrm{pH}$ from the reference $\mathrm{pH}$. The parameter $\alpha$ is the coefficient of proportionality. ISFET readout circuits require that the variation in the threshold voltage be transduced into a form which can be further processed. This is achieved by biasing two of the three parameters of the ISFET (the voltage on the reference electrode $\left(V_{R}\right), V_{D S}$ or $\left.I_{D S}\right)$ with a constant source and reading the third to follow changes in $V_{t h}$. In this paper, the voltage (i.e. $V_{R}$ and $V_{D S}$ ) control and current (i.e. $I_{D S}$ ) readout method is chosen in order to be compatible with DiRIC. Weak inversion operation region is chosen where $I_{I S F E T}$ is exponentially related to the change in $V_{t h}$. The weak inversion provides maximum transconductance efficiency, low power measurement, and the potential for linearisation of current versus the concentration of hydrogen ions [6].

The $I_{D S}$ of an ISFET biased in weak-inversion is then:

$$
I_{D S}=I_{D S 0} e^{\frac{V_{R S}-V_{t h}}{A}}
$$

In which we define $A=n_{I S F E T} \times n \times V_{T}$, where $V_{T}$ is the thermal voltage $K T / q, V_{R S}$ is the voltage difference between the external reference electrode and the source of the ISFET, $n$ is the slope factor of MOSFET in weak inversion, and $n_{I S F E T}$ is the attenuation factor due to the passivation capacitance of the ISFET and depends on the chemical size of the ISFET [10]. The chemical size (i.e. $\left.(W / L)_{\text {chem }}\right)$ of the ISFET is the extended area of the ISFET gate in the last metal layer covered
TABLE II

SiMUlated RESUlTS OF DiRIC

\begin{tabular}{cc}
\hline parameter & value \\
\hline Input current range & $\pm 100 \mu \mathrm{A}$ \\
Input dynamic range & $124 \mathrm{~dB}(3 \mathrm{settings})$ \\
Input referred current noise & $150 \mathrm{pA}$ \\
max input referred leakage & $38 \mathrm{pA}$ \\
Technology & $0.35 \mu \mathrm{m}$ \\
Supply voltage & $3.3 \mathrm{~V}$ \\
Power consumption & $9.9 \mathrm{~mW}$ \\
Area & $0.9 \mathrm{~mm}^{2}$ \\
\hline
\end{tabular}

with the passivation layer that is exposed to the solution and is chosen in this work to be $(W / L)_{\text {Chemical }}=100 / 100 \mu \mathrm{m}$. When $V_{R S}$ and $V_{D S}$ are kept constant, the variation in the threshold voltage (i.e. $\Delta V_{t h}$ ) of the ISFET results in a change of $\Delta I_{D S}$ in the ISFET current.

$$
\ln \left(1+\frac{\Delta I_{D S}}{I_{D S}}\right)=\frac{-\Delta V_{t h}}{A}
$$

For an ISFET in the selected technology (AMS $0.35 \mu \mathrm{m}$ ), the $\alpha$ is approximately $32 \mathrm{mV} / \mathrm{pH}$ [11]. Therefore, a 0.1 unit change in pH leads to $\Delta V_{t h} \simeq 3.2 \mathrm{mV}$. Replacing this into Eq. 3 gives a lower limit on $I_{D S}$. On the other side, assuming $\alpha$ remains constant over the whole $\mathrm{pH}$ range, a 13 unit change in $\mathrm{pH}$ results in maximum $\Delta V_{t h} \simeq 416 \mathrm{mV}$ in Eq. 3 and gives an upper limit on $I_{D S}$. Both $I_{D S, \min }$ and $I_{D S, \max }$ should be in the weak inversion region to ensure the logarithmic behaviour of the ISFET in the whole $\mathrm{pH}$ range. Their values depend on $A$ and subsequently to the electrical and chemical gate size of the ISFET. DC simulations were performed to find the ISFET electrical size and the voltage on the external electrode that satisfies this assuming the minimum-detectable change in current $\left(\min \left(\Delta I_{D S}\right)\right)$ is $0.5 n A$ by the readout circuit. The results are summarized in Table I.

1) Differential ISFET measurement: A REFET that is simply a duplicate of the ISFET is placed in the array to provide an electrochemical reference for the ISFET. The envisaged differential measurement is through two separate chambers each containing one of the ISFET or REFET [12]. To perform a differential $\mathrm{pH}$ measurement, the chamber with the REFET is to be exposed to a solution with a known pH (i.e. 7) while the other chamber is exposed to the solution with unknown pH (i.e. $x$ ).

Since the output of the DiRIC is proportional to the difference in the currents $I_{I S F E T}-I_{R E F E T}$. $V_{R S, R E F E T}$ can be tuned to result in a zero current difference. At this point:

$$
\begin{aligned}
& V_{R S, I S F E T}-V_{R S, R E F E T}=V_{t h, I S F E T}-V_{t h, R E F E T} \\
& \quad=\left.V_{t h, I S F E T}\right|_{p H=x}-\left.V_{t h, I S F E T}\right|_{p H=7}=\alpha(x-7)
\end{aligned}
$$

Therefore, the difference in the $V_{R S}$ voltages of the ISFET and REFET is now linearly proportional to the difference of the $\mathrm{pH}$ in the two chambers. This provides another means to investigate the $\mathrm{pH}$ of the unknown solution and the characteristics of the ISFET. 
TABLE III

COMPARISON WITH DIFFERENTIAL SYSTEMS

\begin{tabular}{cccc}
\hline & {$[8]$} & {$[7]$} & This work \\
\hline On-chip sensors & Yes & No & Yes \\
Fully-integrated readout & No & No & Yes \\
Sample $I_{i n 1,2}$ at same time & Yes & No & Yes \\
\hline
\end{tabular}

To provide an electrical reference for the ISFET, a MOSFET with a similar size is added to the array and its gate voltage is accessible on-chip. The MOSFET can be used to remove the common-mode noise originated in circuits from the ISFET current. Moreover, With a similar argument, the $V_{G S}$ of the MOSFET can be tuned to result in a zero output at DiRIC.

\section{SIMULATION RESULTS}

The simulated input-output characteristics of DiRIC is shown in Fig.3 for different reset clock frequencies. The same clock signal is used in the PGA and the differential amplifier. The input current in Fig. 3 is the current at the input of the main integrator when the input current of the reference integrator is zero. The current range can be divided into three sub-ranges: $0.1 n A$ to $10 n A, 10 n A$ to $1 \mu A$, and $1 \mu A$ to $100 \mu A$. Fig. 3 shows that these sub-range can be covered by only three clock frequencies: $200 \mathrm{~Hz}, 20 \mathrm{kHz}$, and $2 \mathrm{MHz}$. The minimum detectable current is limited by the noise of the system as well as the input-dependant leakage current of the capacitor and the switches of the main integrator. Both effects are simulated for different input current ranges using periodic steady-state simulation and results are reported in Table II.

The circuit is fabricated in $0.35 \mu \mathrm{m}$ technology with four metal layers. The last metal layer is used for the on-chip sensors. The ISFET, MOSFET and REFET are placed on the top and use the passivation layer as the $\mathrm{pH}$-sensitive layer. For other sensors a pad opening layer is used to remove the passivation and make the last metal layer (i.e. Aluminium) exposed for post-processing for the development of electrodes.

DiRIC alone occupies $0.6 \mathrm{~mm}^{2}$, and the sensor array measures $0.7 \mathrm{~mm} \times 0.4 \mathrm{~mm}$. The sensor array is placed in the middle of the chip to ensure more than $0.5 \mathrm{~mm}$ distance from the edges of the fabricated IC. This is to ensure a proper encapsulation of the chip and opening in the sensor area.

\section{CONCLUSiOnS AND FUTURE WORK}

A differential amperometric readout circuit is presented for measurement of on-chip amperometric sensors. Differential readout is achieved through a chain of switched-capacitor circuits followed by an ADC. DiRIC is the first fully-integrated readout circuit that samples input currents at the same clock phase, removes the background current from the sensor current, amplifies the redox current and digitize it (Table III).

The on-chip sensor array includes three sensing sites for metabolite sensing (such as glucose, lactate, and ATP) and an ISFET for $\mathrm{pH}$ measurement. An on-chip $W E_{b k g}$, a REFET and a MOSFET provide the reference for the measurements. The advantage of differential measurement is the elimination

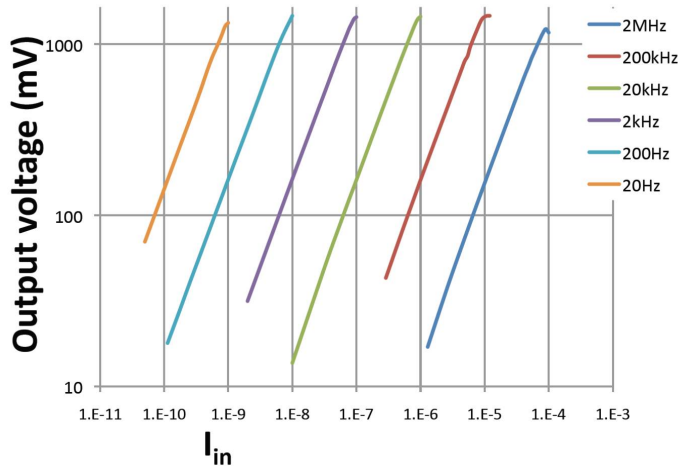

Fig. 3. The $I_{\text {in }}-V_{\text {out }, P G A}$ characteristics of DiRIC for a wide range of input currents at different clock frequencies. Here the gain of PGA is fixed at 1 and the $I_{W E, b k g}$ is zero. The whole range of $100 p A$ to $100 \mu A$ can be covered by three clock frequencies: $200 \mathrm{~Hz}, 20 \mathrm{kHz}$, and $2 \mathrm{MHz}$.

of the background current during the amperometric measurements. Simulation results show that the readout circuit can measure currents in $\pm 100 \mu A$ range maximum input-referred noise of $150 \mathrm{pA}$. The next step is to test DiRIC along with the on-chip sensor array within a micro-fluidic setting for biomolecules and $\mathrm{pH}$ measurement.

\section{REFERENCES}

[1] H. Jafari, K. Abdelhalim, L. Soleymani, E. Sargent, S. Kelley, and R. Genov, "Nanostructured CMOS wireless ultra-wideband label-free pcr-free dna analysis soc," Solid-State Circuits, IEEE Journal of, vol. 49, no. 5, pp. 1223-1241, May 2014.

[2] D. Garner, H. Bai, P. Georgiou, T. Constandinou, S. Reed, L. Shepherd, W. Wong, K. Lim, and C. Toumazou, "A multichannel dna soc for rapid point-of-care gene detection," in Solid-State Circuits Conference Digest of Technical Papers (ISSCC), 2010 IEEE International, Feb 2010, pp. 492-493.

[3] S. Ghoreishizadeh, C. Baj-Rossi, A. Cavallini, S. Carrara, and G. De Micheli, "An integrated control and readout circuit for implantable multi-target electrochemical biosensing," Biomedical Circuits and Systems, IEEE Transactions on, vol. PP, no. 99, pp. 1-1, 2014.

[4] D. L. Robinson, B. J. Venton, M. L. Heien, and R. M. Wightman, "Detecting subsecond dopamine release with fast-scan cyclic voltammetry in vivo," Clinical chemistry, vol. 49, no. 10, pp. 1763-1773, 2003.

[5] S. Carrara, BioCMOS Interfaces and Co-Design. Springer, 2013.

[6] P. Georgiou and C. Toumazou, "Isfet characteristics in cmos and their application to weak inversion operation," SENSORS AND ACTUATORS B-CHEMICAL, vol. 143, pp. 211-217, 2009.

[7] M. Augustyniak, C. Paulus, R. Brederlow, N. Persike, G. Hartwich, D. Schmitt-Landsiedel, and R. Thewes, "A 24x16 CMOS-based chronocoulometric DNA microarray," in Solid-State Circuits Conference, 2006. ISSCC 2006. Digest of Technical Papers. IEEE International, Feb 2006 , pp. 59-68.

[8] A. Hassibi and T. Lee, "A programmable electrochemical biosensor array in $0.18 \mu \mathrm{m}$ standard CMOS," in Solid-State Circuits Conference, 2005. Digest of Technical Papers. ISSCC. 2005 IEEE International, Feb 2005, pp. 564-617 Vol. 1.

[9] K. Martin, L. Ozcolak, Y. Lee, and G. C. Temes, "A differential switched-capacitor amplifier," IEEE Journal of Solid-State Circuits, vol. 22, pp. 104-106, 1987.

[10] L. Shepherd, P. Georgiou, and C. Toumazou, "A novel voltage-clamped cmos isfet sensor interface." IEEE, 2007, pp. 3331-3334.

[11] Y. Liu, P. Georgiou, T. Prodromakis, T. Constandinou, and C. Toumazou, "An extended CMOS ISFET model incorporating the physical design geometry and the effects on performance and offset variation," Electron Devices, IEEE Transactions on, vol. 58, no. 12, pp. 4414-4422, Dec 2011.

[12] M. Kalofonou and C. Toumazou, "A low power sub-uw chemical gilbert cell for isfet differential reaction monitoring," Biomedical Circuits and Systems, IEEE Transactions on, vol. 8, no. 4, pp. 565-574, Aug 2014. 